\title{
UN ENFOQUE DE LA EDUCACIÓN SUPERIOR BAJO UNA NUEVA PERSPECTIVA
}

\author{
Maria Varlene Silva de Oliveira
}

\section{RESUMEN}

\begin{abstract}
Se destaca la importancia de los fundamentos filosóficos de la educación y de la Universidad en el marco de su historicidad. Se Destacan, las tendencias y los cambios que han acosado el sistema superior de América Latina durante su construcción. Sin embargo, se lleva a cabo, un enfoque de los elementos políticos y económicos de la Universidad y, como un ejemplo de la creación y la evaluación de las universidades, explícita de Brasil y Argentina en el contexto de América Latina. Menciona, también, los objetivos y las estrategias de la integración sudamericana, así como las principales transformaciones de la educación superior a través de su historia.
\end{abstract}

Palabras-clave: Universidad. Educación. Hipótesis: filosóficos, políticos y económicos. Integración Sudamericana.

\section{INTRODUCCIÓN}

La educación superior en toda su historicidad se dirigió a través de cambios importantes. Estas transformaciones se dieron en el campo político, filosófico, epistemológico, biológico, ético, pedagógico, económico y psicológico.

Así, este trabajo se propone a examinar solamente los fundamentos filosóficos de la educación y de la Universidad, porque ninguna institución existe por sí mismo, puesto que, necesita estar basada en una filosofía, y eso trae también una ideología propia de su autor, ya sea de carácter puramente público (gobierno) o privada (sin alianzas con el gobierno).

También, podemos preguntarnos cuáles son las tendencias y cambios que enfrenta la Universidad Latinoamericana, revelando las dificultades que se enfrenta a cuenta de estas tendencias.

Aún así, se detallarán los fundamentos políticos y económicos de la Universidad, por ejemplo a través de Brasil y Argentina, el sistema de creación y evaluación de sus ciudades en el contexto de América Latina, porque para que cualquier sistema universitario nazca, es necesario basarse también en la política y la economía que coincide con sus intereses, que 
en la mayoría de las veces no se basa en el bienestar general del hombre, en toda su perspectiva de vida, ya que uno de los objetivos de la Universidad es la de transformar nuestra sociedad en otra mejor, empezando por el ser humano.

Maria Varlene Silva de Oliveira, Graduada en Letras por la Universidad Federal de Maranhão - UFMA, Post Graduada en Lengua Portuguesa por la Facultad Santa Fé - FSF, Mestrado por la Facultad

Interamericana DE Ciencias sociales - FICS- en Ciencias de la Educación varlenematheus@hotmail.com.

Aún así se mencionan, los objetivos estratégicos de la integración

sudamericana, así como las principales transformaciones de la Educación Superior a través de la historia. En este sentido, la Educación como un bien mayor que el hombre tiene el derecho, de construirse por medio de grandes esfuerzos y cambios significativos, porque en la medida en que la tecnología fue surgiendo y modernizándose cada vez más, también la educación tuvo que adecuarse y seguir sus pasos, porque en el mundo de hoy, no hay educación sin tecnología.

De esta manera, la educación superior a través de su construcción ha pasado por un proceso de transformación, especialmente en los aspectos que involucran los campos de la tecnología, evaluación y acreditación. Esta transformación refleja los sistemas educativos en general, porque cualquier reforma en este ámbito, está estrictamente vinculada a las políticas públicas universitarias y, por lo tanto la ideología del gobierno, que en la mayoría de los casos, con respecto a la inversión, así como lo que involucra material humano, no reciben lo que les pertenecen, lo que se convierte en un problema grave para sus desarrollos.

Porque en Brasil, según su legislación y las normas sobre evaluación y acreditación de cursos de nivel superior, la CAPES, junto con el PAIUB, Programa de evaluación Institucional de las Universidades Brasileñas, refieren experiencia.

En esta área, se analizan las innovaciones introducidas por el Examen Nacional de Cursos llamado de "provão", cuya función es la de evaluar los estudiantes de graduación, así como también discutir y resolver los problemas que implica este tema.

Ya Argentina, también trata los mecanismos jurídicos con respecto a la cuestión 
de la experiencia, de la evaluación y acreditación de sus instituciones, principalmente de las Universidades, teniendo como foco la autonomía de estos y, en consecuencia, las críticas se han dirigido a la Educación Superior.

Por lo tanto, este trabajo propone examinar las cuestiones mencionadas en los párrafos anteriores. Una vez que, se analizará, sobre la luz del pensamiento crítico de los autores de este estudio, a través de la óptica científica, filosófica e histórica.

\section{PILARES FILOSÓFICOS, POLÍTICOS Y ECONÓMICOS DE LA EDUCACIÓN UNIVERSITÁRIA}

La educación se refiere a todo tipo de influencias e interrelaciones que convergen en la formación de los rasgos de carácter social y personalidad. Porque el individuo implica una concepción del mundo, ideales, valores, modos de actuar, traduciendo así, sus convicciones ideológicas, morales, políticas, religiosas, es decir, sus principios se reflejan en una acción contra las situaciones reales y los desafíos de la vida cotidiana. Por lo tanto, según (FREIRE, 1988, P.70) "una búsqueda realizada por un sujeto que es el hombre"

De esta manera, la educación de cada sociedad asume una serie de características peculiares, es decir, el hombre se educa para que su vida tenga sentido y significado propio. Como ejemplo de eso, tenemos diferentes sociedades, todas tienen su propia manera de educar, porque la cultura es el factor decisivo, una vez que cada pueblo, cada nación tiene una cultura diferente.

Por lo tanto, durante siglos la acción intencional de educar a la humanidad está siendo modificando, porque esto ha adaptado al hombre a nuevas realidades. En ese sentido, se está haciendo una retrospectiva a través de la historia, a partir de la Edad Media, a principios del siglo XIX, con el advenimiento de la Revolución Industrial, provoca un cambio de acción intencional del hombre de aquella época con una recodificación de la realidad, es decir, con una creación de una nueva realidad, empujándolo a la modernización, pasando este hombre de la sociedad feudal a la burguesía capitalista.

De esta forma, nace una nueva clase social, la burguesía y, en consecuencia, una nueva sociedad, la capitalista, predominante hasta hoy. Esto establece muy bien lo que 
tenemos actualmente, pertenecemos a una sociedad occidental, capitalista, dividida en clases sociales, tales como: la burguesía y el trabajador, en el que la educación también se divide en estas clases. Conforme (WEBER, 1.997, p. 172).

El ámbito de influencia con carácter de dominación sobre las relaciones sociales y los fenómenos culturales es mucho mayor de lo que parece a primera vista. Por ejemplo, es el dominio que se ejerce en la escuela que se refleja en las formas del lenguaje oral escrito considerados ortodoxos. Los dialectos que actúan como lenguaje oficial de las asociaciones políticas autocéfalas, llegaron a ser formas ortodoxas de lenguaje oral y escrito de sus gobernantes, lo que llevó a las separaciones 'nacionales' (por ejemplo, entre Alemania y Holanda). Pero la dominación ejercida por los padres y por la escuela se extiende mucho más Allá de la influencia sobre los bienes culturales (al parecer solamente) hasta la formación del carácter de los jóvenes y con la de los hombres.

Por lo tanto, la educación en su sentido más amplio, debe ser de igual valor para todos, sin distinción de raza, sexo, color, o clase social, aunque esto se materialice en diferentes maneras, con respecto a la cultura.

La educación, sobre todo, es un instrumento que debe velar por la sociedad democrática, occidental, moderna, sostenida por las relaciones de la producción capitalista, la socialización de los conocimientos científicos y tecnológicos, acumulados por la humanidad. En este sentido, la propiedad de los conocimientos organizados por varias ciencias, constituye lo indispensable para la formación y para el ejercicio de la ciudadanía. Como (DURKHEIM, 1.978, P. 41).

La educación es la acción ejercida, por las generaciones adultas, sobre las generaciones que aún no están preparadas para la vida social; tiene como objetivo fomentar y desarrollar, en el niño, cierto número de estados físicos, intelectuales y morales, reclamados por la sociedad política, como un todo, y a través del medio especial que particularmente cada niño sea destinado.

De esta manera, la educación, aún debe aportar, de una manera, las vías de acceso de este mundo complejo y, al mismo tiempo, la brújula que permita navegar a través de ella. Para Morin (1999) la educación debe organizarse en torno a cuatro grandes pilares, 
a lo largo de toda la vida del ser humano, que son los pilares del conocimiento, "aprender a conocer y adquirir los instrumentos de la comprensión; aprender cómo hacer para poder actuar sobre el medio envolvente; aprender a vivir juntos con el fin de participar y cooperar con los otros en todas las actividades humanas; aprender a cómo ser vía esencial que integra los tres precedentes"

En este sentido, la educación en toda su extensión, debe conducir al hombre a revelar su potencial creativo, y esto supone que si se excede la visión puramente instrumental de la educación, considerada como vía obligatoria para obtener resultados (saber hacer, que consiste en la adquisición de diferentes capacidades, con fines de ordenes económicas), pasando a ser considerada en toda su plenitud, a la realización de la persona como un todo.

Por lo tanto, la educación que la Universidad tiene para ofrecer a las sociedades contemporáneas, debe considerar que las nuevas tecnologías cambiarán el concepto del tiempo, del espacio, y la enseñanza, y esto quiere decir, que el individuo tiene derecho a tener una educación que corresponda con su desarrollo integral, porque con eso, el hombre será capaz de vivir en una sociedad tecnológica y globalizada, no sólo cambiando la manera de aprender, pero si lo que necesita saber, utilizando así, su competencia y habilidad.

De esta manera, los pensadores que dilucidaron los fundamentos filosóficos de la educación y de la Universidad, aunque tenían ideas diferentes a lo largo de la historia, todos trataron de definir los principios de modo filosófico que constituya la base de una educación de calidad centrada en la transformación del hombre y, también, la Universidad, donde tiene la oportunidad de construir el conocimiento.

No existe una única fórmula para los pensamientos de estos teóricos, sin embargo, todas sus ideas nos han dejado reflexiones fundamentales para la construcción tanto de la educación y la Universidad, como una institución de educación para todos.(PÉREZ, 2010, P. 74-75) desde el punto de vista ontológico,

Desde el punto de vista ontológico tres principios nos parecen fundadores para la educación: 1) la defensa de la vida; 2) el desarrollo de la libertad, y 3) la formación de la sociedad. Cada uno de estos principios puede terminar una cosmovisión a veces incompatible con las otras. El naturalismo, el vitalismo, el ecologismo o el esencialismo en el primer caso presentarán la educación como una adecuación al orden del Ser. El segundo principio, el de la libertad individual, puede derivar en concepciones individualistas. El tercer principio tiende a 
englobar en la construcción del ser social todas las actividades. Pero si no queremos fragmentar la educación en sus dimensiones particulares, ella debe ser considerada en sus fundamentos vitales, existenciales y sociales.

Por lo tanto, la educación así como también la universidad, sólo pueden ser construidas a través de estos pilares, porque la universidad no existe sin educación. La defensa de la vida, el desarrollo de la libertad, la formación de la sociedad deben ser principios indispensables para la construcción del protagonista de estos dos escenarios, porque la educación y la Universidad sólo fueron construidas sobre la existencia del hombre.

Cabe señalar, que toda la educación es una política, sólo basta necesitamos mirar la historia y la construcción del hombre, que si se da cuenta de este sesgo que la educación se ha construido con el tiempo en una ideología política subordinada, ya sea gubernamental o estado de instancia superior.

Y esto también tiene una base en la economía, de donde provienen las revoluciones y las transformaciones de las sociedades, como se dijo anteriormente. En este sentido los fundamentos políticos y económicos de la universidad están vinculados a través de su propia educación, porque es por su propia construcción trae consigo las características de la economía y de la política, puesto que no existe sin estos elementos.

\section{UNIVERSIDAD LATINO AMERICANA: TRANSFORMACIONES MEDIANTE LA GLOBALIZACIÓN}

Para hablar de la universidad latina americana, hay que considerar algunas condiciones importantes, en el proceso de la educación universitaria y la escolaridad. Entre ellos está la globalización.

Así, el advenimiento de la globalización cambió el comportamiento de todo el mundo en sus estructuras políticas, económicas, sociales y culturales, transformándose en una reestructuración del modo de producción capitalista, lo que significa cambios en la política educacional en varios países, incluidos los de América Latina.

De este modo, la población mundial se encuentra en la tercera revolución tecnológica, centrada en el sector de la microelectrónica, microbiología y en la 
termonuclear. Como consecuencia, esta revolución cambió las formas de ser en la sociedad, abriendo caminos de mayor desarrollo económico y la adquisición de conocimientos. En este sentido, las revoluciones tecnológicas de la modernidad han abierto una amplia gama para la modificación de la producción, de los servicios, de las relaciones sociales y comerciales del mundo.

Así, estos acuerdos han traído grandes beneficios a la población, sin embargo, los peligros para la vida humana llegan a ser más, cada uno dentro de sus especificidades. El ejemplo de esto es la aceleración de la información, ella circula de manera automática, acortando distancias e imposibilitando al hombre en tiempo para codificarlo y aplicarlo a la realidad.

De esta manera, la globalización surgió en el escenario mundial como una forma de sostener y reestructurar el capitalismo para la globalización del capital, teniendo en cuenta el objetivo de llegar a nuevos mercados, es decir, ampliar el mercado de consumo aumentado a través de innovaciones tecnológicas.

La globalización institucionaliza y trata de formar una nueva ideología, de un nuevo orden económico y de política mundial, reduciendo al mínimo la intervención del Estado en el desarrollo de las políticas públicas, el fortalecimiento y manutención de la institución y fuerza coercitiva. Por lo tanto, es un fenómeno que parece sugerir la inclusión de toda la sociedad, pero destaca la exclusión de las regiones y países, especialmente los de tercer mundo.

Siendo así, todo este proceso político y económico ha afectado a la escuela, a la universidad en sus objetivos, prácticas, valores, intereses y necesidades, así como en la forma de atender a los intereses del mercado. Y en este sentido, la educación se convierte apenas en un medio de preparación para satisfacer las demandas del mercado y del mundo globalizado. Para (LIBÂNEO, 2003, P.76) el capitalismo es:

Guiado por la ideología del libre mercado. Rompiendo las fronteras y debilitando los gobiernos, hace que los mercados se unan y se dispersen al mismo tiempo se impone la lógica de la exclusión, observada en el mundo de la producción, del comercio, del consumo, de la cultura, del trabajo y de las finanzas.

Por lo tanto, el Estado neoliberal viene desobligándose de la educación, especialmente de la educación universitaria. Porque con la exigencia del mercado, el 
trabajador necesita adquirir nuevas destrezas e capacidades para monitorear el avance científico-tecnológico de las empresas, desde entonces, su orientación, forma los consumidores sofisticados y competentes.

La ideología del Banco Mundial en relación con la educación se refleja en las tendencias del nuevo orden mundial, que en las palabras de (LIBÂNEO, 2003 P. 1003) quiere decir que "requiere que la educación sea articulada en el nuevo paradigma productivo para garantizar el acceso a los nuevos códigos de la modernidad capitalista.”

Se entiende, entonces, que toda la política educativa está centrada, sobre todo en las necesidades del mercado, del nuevo paradigma productivo y neotecnicismo.

Basándose en todos estos supuestos, tanto la escuela como la universidad como instituciones educativas, están siendo interrogados acerca de su papel frente a las transformaciones económicas, políticas, sociales y culturales del mundo contemporáneo, teniendo en cuenta que emplean, sobre todo, los avances tecnológicos, la reestructuración del sistema de producción y desarrollo, el papel del Estado, las modificaciones a su funcionamiento y los cambios en el sistema financiero. (PÉREZ, 2010 p. 36) detalla las tendencias que reprochan tanto la universidad coma a la educación.

La globalización impulsa la internacionalización de los procesos educativos y crea la posibilidad de pensar en la preparación de los individuos para una responsabilidad planetaria. (...) La informatización de la sociedad genera nuevas intersubjetividades virtual, facilita el acceso directo a todas las fuentes de información científica y cultural, favorece el auto aprendizaje, exige mayor capacidad de reflexión, disminuye los hábitos de la lectura y la escritura; (...) La revalorización de las identidades étnicas; (...) La extensión de la pobreza y la exclusión de vastos sectores sociales; (...) El surgimiento de una economía que tiene al conocimiento como factor principal lleva a prestar más atención a la formación científica y técnica de los individuos; (...).

De esta manera, la escuela, y la universidad han sido afectadas de diversas maneras, aún así, han intentado cambiar para satisfacer las nuevas perspectivas de la sociedad actual, es decir, tienen que satisfacer las necesidades de una sociedad pluralista, de cultura uniforme y grandes avances tecnológicos.

En este sentido, estas dos tendencias, el Neoliberalismo y la Globalización, se adaptan según las circunstancias de la sociedad, teniendo en cuenta el instituto de sustentar 
ideológicamente determinados procesos de modernización de la historia. Estas dos tendencias consisten en el paradigma de la libertad económica, de la eficiencia, de la calidad y de la igualdad. (LIBÂNEO, 2003 P.91) corrobora.

Los defensores del liberalismo social creen que, a través de la universalización de la educación, sería posible establecer las condiciones para que la institución de la sociedad democrática, moderna, científica, industrial y completamente desarrollada. La ampliación cuantitativa de acceso a la educación garantiría la igualdad de oportunidades, el máximo de desarrollo individual y la adaptación social de cada uno conforme su inteligencia y capacidad.

Justamente, las organizaciones multilaterales, tales como (Banco Mundial, UNESCO y otros) difundirán la educación y la producción del conocimiento como un nuevo proceso de producción y, por lo tanto, la educación se convierte como una modalidad que tiene la función para producir más y mejor, todo a favor del sistema capitalista.

En este sentido, los objetivos estratégicos de la integración Suramericana consiste en la transformación de la educación, con miras a aumentar la capacidad del potencial científico-tecnológico, así como la formación de una nueva sociedad, vinculada a la democracia y a la igualdad, es en realidad la ideología de la globalización.

\section{EJEMPLO DE LA EDUCACIÓN SUPERIOR EN ALGUNOS PAÍSES DE AMÉRICA LATINA}

Para tratar las diferencias públicas de acreditación de Brasil y Argentina, es necesario analizar y entender un poco de sus historicidades, así como las principales características distintivas de la educación superior que los forman.

Brasil, a pesar de tener una tasa más alta cuando se restringe a sus estudiantes (1,5 millones contra 1 millón en Argentina), el número de estudiantes en este país en relación con la población es mayor. La historicidad de estas tasas, por lo menos en 1980, se acercó. Como se puede ver en la siguiente tabla:

Matrícula en la Educación Superior por 10 mil habitantes, Brasil, Argentina y la razón, 1950/1990

\begin{tabular}{|lr|r|r|r|r|r|}
\hline País & Año & 1950 & 1960 & 1970 & 1980 & 1990 \\
\hline
\end{tabular}




\begin{tabular}{|l|r|r|r|r|r|}
\hline Brasil & 10,0 & 13,2 & 45,7 & 93,7 & 104,8 \\
\hline Argentina & 50,0 & 84,4 & 122,4 & 172,6 & 293,9 \\
\hline Razón Argentina/Brasil & $5,0 / 1$ & $6,1 / 1$ & $2,7 / 1$ & $1,8 / 1$ & $2,8 / 1$ \\
\hline
\end{tabular}

Fuente: Prior (1995) y Chiroleu (1996).

La razón, de esta diferencia se debe probablemente a la reanudación de las políticas públicas universitarias que favorecen la entrada de los alumnos sin restricción en las Universidades Argentinas, esto por cuenta del fin de la dictadura militar. Con eso, hubo también el crecimiento de las instituciones de Educación Superior tanto públicas como privadas.

Por lo tanto, la construcción de las Universidades da Argentina y Brasil han sido momentos decisivos y diferentes en relación con las políticas públicas para la acreditación. En Brasil, después de intentos fallidos a principios del siglo XX, la Universidad de Rio de Janeiro, ha tenido éxito. Argentina, también, pasó por varios problemas en la formación de sus Universidades. La Universidad de Córdoba, fue una de las pioneras, fundada en 1613 pelos Jesuitas, reconocidos sólo en 1622, continuada a ser administrada por gestión administrativa. Cuña $(1998$, p.18) hace explícito lo que se analiza.

L institución universitaria es relativamente reciente en Brasil, aunque la Educación Superior ya existía en el siglo XXI, en los colegios jesuitas (Bahía, 1572), luego de varios intentos fallidos a principios del siglo $X X$, la primera Universidad exitosa fue en 1920 la Universidad de Rio de Janeiro (después la Universidad de Brasil, luego la Universidad de Rio de Janeiro). (...) Argentina tuvo su primera Universidad de Córdoba, fundada en 1613 por los jesuitas, reconocida por el Decreto Real en 1622, pero continúa bajo gestión clerical hasta ser nacionalizada en 1856.

Actualmente, en Brasil, la Educación Superior se desarrolla desde el siglo XIX, es decir, primero con instituciones aisladas y después con las Universidades creadas por facultades públicas o privadas. Ya en Argentina, en 1970, presentaron un alto crecimiento de instituciones no universitarias privadas, a pesar de las universidades sean instituciones propias de la educación superior.

Por lo tanto, Brasil presenta en el ámbito universitario su prestigio a través de la Educación Superior, conduciendo en este caso, la conciencia social a cualquier institución, cursos, profesores y estudiantes. Em Argentina, a su vez, no existen límites entre las 
Universidades y las instituciones terciarias que son muy claras tanto en la legislación como en la conciencia social, por eso resulta difícil llevar a cabo las estadísticas oficiales sobre el número de alumnos en las instituciones no universitarias, esto significa tanto para las públicas como las privadas.

Bajo la óptica brasileña, este contexto no presenta un entendimiento muy claro, con la excepción de la comparación de estudios de posgrado latu sensu. Son en estas instituciones donde se forman la mayor parte del grupo de profesores de nivel primario, secundario y una porción de la parte superior.

En este sentido, la Educación Superior en Brasil se convierte más dispersos, ya que el poder político de la clase elitista y estándar más descentralizado del poder económico. Mientras, en Argentina, principalmente en Buenos Aires, la centralidad económica es evidente.

\section{LAS POLÍTICAS PÚBLICAS COMO PRERREQUISITO IMPORTANTE EN LA ENSEÑANZA SUPERIOR}

En algunos países de América Latina, como en Brasil, según la ley brasileña, los centros universitarios son creados a partir de facultades integradas, a las facultades propias, de los institutos de enseñanza superiores que demuestran excelencia en la enseñanza. Por lo tanto, estas universidades serán evaluadas cada cinco años a los efectos de la acreditación. No ocurre lo mismo con las facultades aisladas o integradas, con los institutos superiores y escuelas superiores, que según el Consejo Nacional de Educación (CNE), están autorizados a operar en diferentes plazos.

Sin embargo, esta situación cambia, cuando estas instituciones son nuevas, estas comienzan a ser acreditadas por medio del acto jurídico de autorización para que sus cursos puedan funcionar, a través del resultado de la deliberación del Consejo de Educación Superior del Consejo Nacional de Educación, aprobada por el Ministro de Educación. De esta manera, el periodo de validez de la autorización de estas nuevas instituciones es de dos o tres años. Esto vale para los cursos de duración de cuatro o cinco años.

Sin embargo, cuando el término de validez termina, se da una nueva evaluación, in loco, teniendo en cuenta el reconocimiento y la renovación de la 
autorización, por lo tanto, el período de validez de la acreditación es de cinco años. Según Cunha (1998, p.27), hay un tiempo definido para el reconocimiento de tales instituciones.

El reconocimiento (por primera vez y la renovación periódica del reconocimiento) de los cursos y de las calificaciones de cualquier institución de nivel superior puede ser solicitado desde el segundo o tercer año de funcionamiento. El Consejo de Educación Superior del CNE se pronunciará sobre la petición después del informe de la visita de la comisión de expertos, elaborado sobre los estándares, criterios e indicadores de calidad de los cursos y áreas específicas, establecidos por SESU/MEC, y oída por CES/CNC.

Si embargo, en el caso de las instituciones con autonomía, la creación o extinción de cursos de graduación y posgrado es libre, así como la expansión y reducción del número de vacantes, debido a que no requiere la solicitación previa de autorización. Ya las que no tienen este privilegio, necesitan solicitar la autorización de sus cursos para el Ministerio de Educación. Para la correcta ejecución de la creación de estos cursos, estas instituciones pasan por una burocracia, es decir, sólo después de análisis de los proyectos de verificación sobre el terreno y la evaluación es que se deberán enviar los informes al Consejo Nacional de Educación, para la deliberación, que todavía depende, de la aprobación del ministro.

En lo referente con la creación de los cursos de Medicina, de Odontología y de Psicología, es indispensable la apreciación del Consejo Nacional de la salud para cualquier institución.

A pesar de ello, vale la pena señalar, que las universidades privadas se crean a partir de las instituciones existentes, ya que, para esta creación, es necesario enviar al Ministerio de la Educación es un proyecto que proporciona una revisión y, posteriormente, al Consejo Nacional de Educación junto con el resultado de las evaluaciones realizadas. Ya las públicas se crean como determinación legal, no requieren una pronta solicitación al Consejo Nacional de Educación.

En el caso de Argentina, con respecto al sistema de acreditación d las universidades públicas y privadas, que también se forman a través de la ley Federal de Educación, se centran en una evaluación continua y sistemática del sistema educativo. Basado en esta hipótesis, la Ley de Educación dedica su compromiso a la educación superior. Porque determina que el Ministerio de la Cultura y de la Educación y todos los 
demás órganos relacionados con el buen funcionamiento de la misma, sobre todo la enseñanza de las Universidades, que deben asegurar la formación de calidad de los ciudadanos en su conjunto, teniendo en cuenta la evaluación y acreditación de cursos de control de estas universidades.

Con respecto a la solicitud de acreditación de cursos, es fundamental destacar que aunque la Ley de Educación Superior no apunta a una necesidad de evaluación específica de las mismas, aunque sí, a priori, los cursos deben cumplir con los requisitos mínimos y los cumplimientos exigidos por esta ley. De lo contrario, la Comisión Nacional de Evaluación y Acreditación, pueden optar por intervenir en la suspensión de la inscripción de nuevos estudiantes, con el objetivo de corregir las deficiencias encontradas sin poner en peligro los derechos de los estudiantes matriculados.

A través del análisis realizado sobre las diferencias y similitudes de las políticas públicas de la acreditación de Argentina y Brasil, se puede observar que ambos países tienen cualidades y deficiencias en el sistema de educación superior.

Por lo tanto, las Universidades brasileñas y las argentinas han experimentado retos históricos, lo que las hace especialmente próximas y, al mismo tiempo, lejanas. Esta dicotomía se explica por su propia construcción histórica de estas instituciones, que aunque hayan surgido a partir del siglo XVI (Brasil, 1572 y Argentina, 1613). Tanto en un contexto del siglo XXI, todavía sigue experimentando desafíos considerables, tales como: la búsqueda por mejorar la calidad de los cursos, así como el mantenimiento del equilibrio de las relaciones de la oferta y la demanda.

En lo referente a estructuras de la universidad, se nota algo interesante. En Brasil, las Universidades se encuentran dispersos geográficamente, mientras que en Argentina, las principales universidades se concentran en Buenos Aires. Por lo tanto, con respecto a esta cuestión, uno tiene que preguntarse, si los parámetros de asistencias de la Educación Superior (Brasil/Argentina) cumplen con las expectativas, o hasta que punto estas causas (concentración / dispersión de las unidades de enseñanza superior) pueden influir en las políticas públicas de la acreditación universitaria, teniendo en cuenta, que aunque la universalización de la educación superior sea algo prioritario en las agendas de las políticas gubernamentales, aún así, no está claro la armonía entre los desafíos regionales y los cursos ofrecidos por estas instituciones. 
Por otro lado, el modelo Argentino, con respecto a la autonomía de gestión, que nos parece ser lo más razonable, debido a la existencia de una mayor libertad en el control de las políticas de investigación y extensión universitaria, lo que de una manera también significa en medir las necesidades de inversiones, en soluciones científicas y tecnológicas. En el caso de Brasil, esta es una realidad que presenta serios desafíos, ya que el gobierno mantiene el control de las universidades públicas y mantiene una supervisión en las instituciones privadas. Sin embargo, esta opción del gobierno brasileño, a fin de proporcionar una educación superior pública y de calidad, no pasará por el paternalismo simple, sin embargo, por encima de todo, es un derecho garantizado por la Constitución brasileña de 1988.

A este aspecto, cabe mencionar que Brasil se mantuvo vinculado a su formato de las políticas relacionadas con la gestión de las universidades públicas, en detrimento de las directrices del Banco Mundial, en el que los gobiernos de Brasil y Argentina se proponen la transferencia de las redes públicas de educación al sector privado.

En este sentido, cabe mencionar, que las orientaciones del Banco Mundial, Se encontrarán en la política universitaria argentina cierta conformidad, teniendo en cuenta que las cobranzas de anualidades, ya es una realidad practicada y prevista, incluyendo en ley.

\section{CONSIDERACIONES FINALES}

A través del análisis realizado, se observó que el sistema universitario dentro del proceso educativo, en el curso de la historia ha sufrido una gran transformación para comenzar por el acceso, porque en los tiempos antiguos, cursando un curso superior, era casi imposible, especialmente para las personas de clase baja. El ejemplo de esta facilidad ha sido la creación de los sistemas universitarios virtuales, que ofrece diversos cursos.

Este avance significativo hizo posible la realización del sueño de millones de personas. La pluralidad de las instituciones, sea privada o pública, han contribuido para el aumento de la gran demanda de personas ingresando en la universidad, así como la opción del curso en el que tiene como objeto de estudio.

Por lo tanto, los programas de inclusión universitaria en Brasil, fueron creados, 
para los Estudiantes, que salen del secundaria, pasando los pasos propuestos por el programa, pueden ingresar en el curso escogido en una universidad, esto para las universidades públicas. Uno de ellos ENEM.

De esta manera, los actuales gobiernos están invirtiendo mucho más en recursos tecnológicos para la difusión del conocimiento en los sistemas universitarios, teniendo en cuenta que, en la actualidad, en general, la sociedad ha sido transformada a través de la conciencia de la importancia de buscar el conocimiento, especialmente en los centros de las universidades.

Por lo tanto, ni siquiera por las grandes transformaciones a lo largo de la historia, la universidad ha buscado éxito, cuando lo logra, a pesar de los males del sistema capitalista, convirtiendo al hombre en una persona crítica, capaz de cambiar todo su seno social en el que vive y, todavía, cambiar su sociedad, porque el conocimiento es la única arma que tiene la capacidad para dar rienda suelta al hombre, volviéndolo verdaderamente libre.

En pocas palabras, las universidades, a través de sus construcciones que involucran aspectos de acreditación, evaluación, filosóficos, sociológicos y sobre todo políticos pasaron por un momento decisivo, aunque esto ha contribuido para a importantes avances de estos aspectos. En el caso de Brasil y Argentina, tienen rasgos comunes y diferentes situaciones. Ambos ya han tenido experiencias de restricción de la libertad intelectual. Y, este silencio impuesto por la dictadura, se convirtió en una voz altiva y defensora de los intereses de la libertad intelectual, la conquista de nuevas herramientas y de la expansión del conocimiento de la contemporaneidad.

\begin{abstract}
The importance of the philosophical foundations of the education is pointed out and of the University in the context of your historicidade. Detach-se tendencies and exchanges that it has been pursuing the Latin superior system American during your construction. He/she/you takes place, still, an approach of the political and economical foundations of the University. it is mentioned, also, the objectives and strategies of the integration American sula, as well as the main transformations of the superior education through your history.
\end{abstract}

Key-Words: University. Education. Foundations: philosophical, political and economical. American South integration. 


\section{REFERENCIAS}

DURKHEIM, E. Educación y Sociología. 11. Ed. São Paulo: Melhoramentos, 1978.

FREIRE, P. Educación y Cambios. 24. ed. Rio de Janeiro: Editora Paz e Terra, 2001.

LIBÂNEO, José Carlos ET al. Educación y Escuela: políticas, estructura y organización. São Paulo: Córtex, 2003.

PÉREZ, Augusto Lindo. Universidad: conocimiento y reconstrucción social. - $1^{\mathrm{a} e d .-}$ Buenos Aires: Biblos, 2003.

PÉREZ, Augusto Lindo. ¿Para qué educamos hoy? : Filosofía y teoría de la educación.$1^{a}$ ed.- Buenos Aires: Biblos, 2009.

WEBER, M. Biografía de Max Weber. México, Fondo de Cultura Económica, 1997.

VEGA, Roberto 1. La gestión de la universidad: planificación. Estructuración y control 1 ED- Buenos Aires: Biblos, 2009.

VELLOSO, Jacques. La educación superior y el Mercosur. Org. Rio de Janeiro: Garamond. 1998. 